\title{
Nutrient Control of Energy Homeostasis via Gut-Brain Neural Circuits
}

\author{
Gilles Mithieux \\ Inserm U-855, Faculté de Médecine Lyon-Est 'Laennec', and Université de Lyon, Lyon, and Université Lyon 1, \\ Villeurbanne, France
}

\section{Key Words}

Gastrointestinal nerves · Intestinal gluconeogenesis .

Food intake - Endogenous glucose production - Obesity . Diabetes

\begin{abstract}
Intestinal gluconeogenesis is a recently described function in intestinal glucose metabolism. In particular, the intestine contributes around $20-25 \%$ of total endogenous glucose production during fasting. Intestinal gluconeogenesis appears to regulate energy homeostasis via a neurally mediated mechanism linking the enterohepatic portal system with the brain. The periportal neural system is able to sense glucose produced by intestinal gluconeogenesis in the portal vein walls, which sends a signal to the brain to modulate energy and glucose homeostasis. Dietary proteins mobilize intestinal gluconeogenesis as a mandatory link between the sensing of these proteins in the portal vein and their wellknown effect of satiety. Comparably, dietary soluble fibers exert their antiobesity and antidiabetic effects via the induction of intestinal gluconeogenesis. Finally, intestinal gluconeogenesis might be involved in the rapid metabolic improvements in energy homeostasis induced by gastric bypass surgeries of obesity.

(c) 2014 S. Karger AG, Basel
\end{abstract}

\section{Introduction}

The worldwide increase in obesity and associated pathologies urges researchers to seek a better understanding of the mechanisms of control relating to food intake and energy homeostasis. The sensations of hunger and fullness are key determinants in the control of food intake. In normal-weight individuals, there is a balance between the sensation of hunger preceding a meal and the sensation of fullness after nutrient absorption. This balance is deregulated in obesity, resulting in the sensation of fullness being inappropriately delayed or blunted [1]. The mechanisms underlying the shift from sensation of hunger to sensation of fullness after meals include the modulation of gastric distension, changes in gut motility and the secretion of gastrointestinal hormones such as ghrelin, cholecystokinin, peptide $\mathrm{YY}_{3-36}$ and glucagon-like peptide 1 [2]. It is generally considered that these effects occur only after changes in circulating gastrointestinal hormone, the levels of which are detected by the hypothalamus [3-6]. However, the gastrointestinal neural system plays a key role in the hunger-curbing effect of gastric distension. The latter is sensed by baroreceptors present in the nerves of the stomach walls and is transmitted to the brain by the ventral vagus nerve [2]. There is also ev-

\section{KARGER}

E-Mail karger@karger.com

www.karger.com/nen (c) 2014 S. Karger AG, Basel

0028-3835/14/1003-0089\$39.50/0
Gilles Mithieux

UMR Inserm U-855, Faculté de Médecine Lyon-Est 'Laennec'

7-11 rue Guillaume-Paradin

FR-69372 Lyon (France)

E-Mail gilles.mithieux@inserm.fr 
idence that glucose, a major macronutrient, can regulate hunger and food intake via activation of peripheral nerves [7].

\section{Gluconeogenesis Is a New Intestinal Metabolic Function}

It is well documented that the gut can participate in the regulation of glucose homeostasis via its capacity to uptake and metabolize glucose [7]. In the 1970s, using a new model of isolated perfused intestine in the rat, Windmueller and Spaeth $[8,9]$ showed that the small intestine (SI) is able to utilize glutamine with the same efficiency as glucose. Glutamine was previously considered only as a major energy substrate for the SI, but it is now shown that the glucose-6-phosphatase (Glc6Pase) gene is expressed in the rat and human SI, i.e. the gene conferring gluconeogenesis function to the liver and kidney [10]. This report documented a key connection between glutamine and glucose intestinal metabolisms. Basically, it showed that, like the liver and kidney, the SI is able to contribute to endogenous glucose production via gluconeogenesis. In particular, this change takes place during fasting or under insulin deficiency conditions, such as streptozotocin-induced diabetes [11], and glutamine is the major precursor of glucose formed in the SI during fasting [11]. Interestingly, both Glc6Pase and phosphoenolpyruvate carboxykinase-cytosolic forms (PEPCK-C) are controlled by insulin at the level of gene expression both in the SI [10-13] and the liver [14-16]. Biochemical mechanisms of regulation of Glc6Pase activity also exist and are dependent either on insulin signaling $[17,18]$ or on nutrients [19-22]. Intestinal glucose production is rapidly inhibited by insulin [11]. Therefore, short-term regulations of Glc6Pase activity may take place in the SI. This is an issue that requires experimental consideration. It is interesting to mention that the expression of gluconeogenesis enzymes in the SI has been confirmed in several species, including trout [23], rat [24] and human [25]. It has also been suggested that intestinal glucose production may take place during the anhepatic phase of liver transplantation in humans [26].

\section{Sensing of Glucose in the Portal Vein Regulates Food Intake}

It has long been hypothesized that glucose might regulate hunger in the course of meal digestion. In line with this rationale, seminal studies reported that glucose infu- sions into the portal vein at a rate equivalent to, or slightly higher than endogenous glucose production, decrease food intake in previously fasting rats $[27,28]$. Portal glucose infusions initiated various physiological and behavioral responses, including acquisition of food preference [29], activation of vagal nerves [30], and activation of neurons in the nucleus of the solitary tract [31] and in the hypothalamus [32, 33]. However, glucose infusions at much lower rates are sufficient to initiate both the activation of hypothalamic nuclei and a limitation of food intake in rats $[34,35]$. Since intestinal gluconeogenesis releases glucose in the portal vein, this led to the hypothesis that intestinal gluconeogenesis could regulate food intake via portal glucose sensing. Recently, the molecular mechanism was described for the portal sensing of glucose. A body of evidence allowed us to suggest that the sodium-glucose cotransporter 3 (SGLT3) could be responsible for portal glucose sensing-initiated events, rather than the glucose transporter Glut2, as generally hypothesized [35] (fig. 1).

\section{Induction of Intestinal Gluconeogenesis and Protein-Induced Satiety}

It has long been known that dietary proteins exert potent satiety effects, but the underlying mechanisms were not understood. In fact, it has been shown that proteinenriched diets (PEDs) initiate their satiety effects indirectly via intestinal gluconeogenesis and portal glucose sensing. PEDs induce the expression in the gut of genes regulating gluconeogenesis. This results in release of glucose into the portal vein in the postabsorptive period that amounts to $20-25 \%$ of endogenous glucose production [34]. This is sufficient to activate the portal glucose sensor and to curb hunger and food intake. The portal ascending nerves are essential in this phenomenon. This effect of PEDs is lost in mice deficient for intestinal Glc6Pase, which demonstrates the causal relationship between intestinal gluconeogenesis and the satiety effect deriving from food protein [36]. The mechanism by which PEDs were able to induce intestinal gluconeogenesis gene expression has been recently elucidated. In the brain, $\mu$-opioid receptors (MORs) can interfere with the control of food intake via their role in the 'reward' system [37]. Previous studies showed that various proteolytic moieties released from ingested protein exhibit $\mu$-opioid activity in vitro [38-41]. Also, peptides derived from the digestion of protein could be delivered into the portal blood after crossing the enterocyte mucosa [42]. This has raised the attractive hypothesis that MOR present in the portal vein
Mithieux 


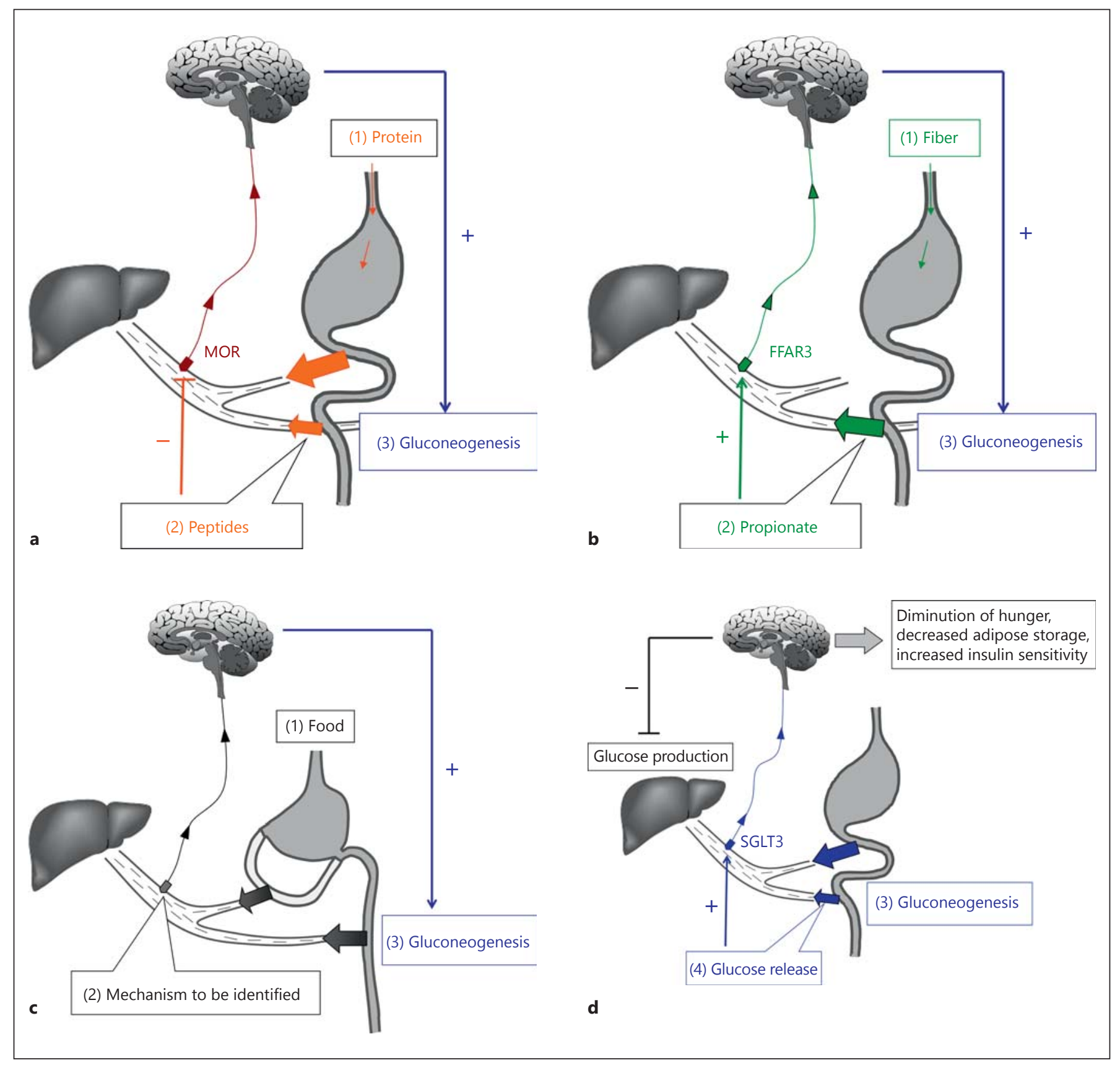

Fig. 1. Mechanisms underlying the regulation of intestinal gluconeogenesis (IGN) and its central and metabolic effects. a The ingestion of dietary protein (1) is followed by the appearance in the portal vein of peptides that antagonize MOR present in the portal vein nerves (2), which sends a signal to the brain. In response, IGN is activated (3). b Following the ingestion of dietary soluble fiber (1), SCFAs are produced from microbiotal fermentation in the distal gut. Propionate released in the portal vein activates FFAR3 present in the portal nerves (2), which sends a signal to the brain. IGN is activated in response to this signal (3). c After gastric bypass surgery, nutrients released directly in the distal gut activate IGN by a mechanism dependent on the integrity of the portal vein nerve that remains to be identified. $\mathbf{d}$ On activation of gluconeogenesis (3), glucose released into the portal vein binds to and activates SGLT3 (4), signaling to the brain regions controlling energy homeostasis, including the hypothalamus. The metabolic benefits are multiple: inhibition of hepatic glucose production, decreased hunger, decreased adipose storage, increased insulin sensitivity (particularly at the level of hepatic glucose production). 
nerves might be involved in the control of food intake by protein via intestinal gluconeogenesis. Comparing the effect of MOR agonists, MOR antagonists or various peptides, we showed that peptides behave as MOR antagonists to induce intestinal gluconeogenesis and that this MOR-dependent induction of intestinal gluconeogenesis genes (resulting from an efferent nervous signal from the brain) might account for the decreased hunger promoted by PEDs [43] (fig. 1). In addition, MOR-knockout mice are insensitive to PED and mice with intestinal Glc6Pase deletion do not decrease food intake in response to portal infusions of MOR antagonists or peptides [43].

\section{Antiobesity and Antidiabetic Effects of Dietary Fiber and Intestinal Gluconeogenesis}

Dietary fiber is a key component of healthy food, encompassing fruit and vegetables. Unlike insoluble fiber (as cellulose), soluble fiber (as fructo-oligosaccharides) is fermented by microbiota in the distal gut to produce short-chain fatty acids (SCFAs): acetate, propionate and butyrate, which are used in metabolism by the host. Fiberenriched diets are known to improve insulin sensitivity and glucose tolerance in lean and obese diabetic subjects [44]. However, as for PED, the mechanisms underlying the metabolic benefits associated with soluble fiber remained elusive. These benefits were thought to be mediated by SCFAs through regulation of whole-body energy homeostasis. Because propionate is a possible substrate of gluconeogenesis, we raised the possibility that intestinal gluconeogenesis and portal glucose sensing contribute to the metabolic benefits of soluble fiber [45].

We have shown that propionate is a precursor of glucose in the intestine [45]. There is also a strong induction of the regulatory gluconeogenesis genes Glc6Pase and PEPCK-C in the jejunum of rats fed a diet enriched in either fructo-oligosaccharides, propionate or butyrate. Interestingly, the induction of gluconeogenesis gene expression takes place in the colon, where microbiota reside. At a mechanistic level, butyrate stimulates the expression of gluconeogenesis genes directly in the enterocyte mucosa via an intracellular cAMP increase, the latter being a key factor influencing intestinal gluconeogenesis gene expression [46]. In contrast, propionate acts via a portalhypothalamic neural circuit initiated by the activation of the free fatty acid receptor FFAR3 to increase intestinal gluconeogenesis gene expression [45] (fig. 1).

As expected feeding of soluble fiber or SCFAs is related to several metabolic benefits for rats. These include a moderation of body weight gain and a decrease of fat depots deriving from increased energy expenditure, and a better insulin tolerance and glucose tolerance resulting in a lowering by $10-15 \%$ of fasting plasma glucose. In agreement with a causal role of intestinal gluconeogenesis and portal glucose sensing in these metabolic improvements, the benefits are strictly dependent on the integrity of the periportal nervous system in rats and are absent in mice with a deletion of intestinal Glc6Pase [45].

\section{Intestinal Gluconeogenesis in Gastric Bypass}

Unlike gastric banding, gastric bypass surgery produces dramatic benefits on body weight and glucose homeostasis in morbidly obese diabetic patients [47]. It is generally believed that enhanced secretion of glucagon-like peptide 1 in response to a meal could account for the benefits of gastric bypass [47]. However, using a model of gastroenteroanastomosis (promoting metabolic benefits comparable to those of gastric bypass in mice), we observed that a marked induction of intestinal gluconeogenesis relayed by portal glucose sensing occurred in obese mice with diabetes after gastric bypass, and not after gastric banding [48] (fig. 1). No metabolic benefits of bypass occurred after inactivation of periportal nerves, suggesting a key role of portal glucose sensing [48]. Interestingly, the question of intestinal gluconeogenesis has been raised recently in relation to gastric bypass surgery in humans [49]. It is strongly suggested that, intestinal glucose release may take place during the postabsorptive period after gastric bypass surgery in obese humans [49, 50]. It must also be noted that data from our study in mice [48] have recently been supported by a similar study of bypass surgery in the Goto-Kakisaki diabetic rat [51]. Finally, it must be emphasized that intestinal gluconeogenesis curbing hunger sensation and improving insulin sensitivity, and glucagon-like peptide 1 secretion improving insulin secretion, may act in synergy to promote the whole beneficial effect of gastric bypass surgeries on glucose and energy homeostasis [52].

\section{Conclusions}

The gut has long been known as a high glucose consumer. Recently, the role of intestinal gluconeogenesis in the control of energy homeostasis through portal glucose sensing and a communication with the brain was described. Dietary proteins mobilize intestinal gluconeo-
Mithieux 
genesis as a mandatory link between their detection in the portal vein (depending on peptide sensing by MOR) and their effect of satiety. In the same manner, dietary soluble fibers exert their antiobesity and antidiabetes effects via the induction of intestinal gluconeogenesis. FFAR3 is a key neural receptor involved in the specific action of propionate to activate a gut-brain reflex arc triggering the induction of the gut gluconeogenesis genes. Relating to the mechanism of glucose sensing from intestinal 'gluconeogenic' origin, the role of the glucose receptor SGLT3 in the periportal nerves has been strongly inferred. Finally, intestinal gluconeogenesis might also be involved in the rapid metabolic improvements induced by gastric bypass surgeries of obesity. Thus, the knowledge of intes- tinal gluconeogenesis and its dialog with the brain might pave the way to future approaches of prevention and/or treatment of metabolic diseases.

\section{Acknowledgments}

The author would like to thank all the members of his team, who have participated over several years in the work summarized here.

\section{Disclosure Statement}

The author declares no conflict of interest in relation with this article.

\section{References}

1 Little TJ, Feinle-Bisset C: Effects of dietary fat on appetite and energy intake in health and obesity: oral and gastrointestinal sensory contributions. Physiol Behav 2011;104:613-620.

$\checkmark 2$ Janssen P, Van den Berghe P, Verschueren S, Lehmann A, Depoortere I, Tack J: Review article: the role of gastric motility in the control of food intake. Aliment Pharmacol Ther 2011; 33:880-894

3 Date Y, Murakami N, Toshinai K, Matsukura $\mathrm{S}$, Niijima A, Matsuo $\mathrm{H}$, et al: The role of the gastric afferent vagal nerve in ghrelin-induced feeding and growth hormone secretion in rats. Gastroenterology 2002;123:1120-1128.

-4 Smith GP, Jerome C, Cushin BJ, Eterno R, Simansky KJ: Abdominal vagotomy blocks the satiety effect of cholecystokinin in the rat. Science 1981;213:1036-1037.

5 Abbott CR, Monteiro M, Small CJ Sajedi A, Smith KL, Parkinson JR, et al: The inhibitory effects of peripheral administration of peptide YY(3-36) and glucagon-like peptide-1 on food intake are attenuated by ablation of the vagal-brainstem-hypothalamic pathway. Brain Res 2005;1044:127-131.

6 Vahl TP, Tauchi M, Durler TS, Elfers EE, Fernandes TM, Bitner RD, et al: Glucagon-like peptide-1 (GLP-1) receptors expressed on nerve terminals in the portal vein mediate the effects of endogenous GLP-1 on glucose tolerance in rats. Endocrinology 2007;148:49654973.

7 Delaere F, Magnan C, Mithieux G: Hypothalamic integration of portal glucose signals and control of food intake and insulin sensitivity. Diabetes Metab 2010;36:257-262.

8 Windmueller HG, Spaeth AE: Uptake and metabolism of plasma glutamine by the small intestine. J Biol Chem 1974;249:5070-5079.

-9 Windmueller HG, Spaeth AE: Identification of ketone bodies and glutamine as the major respiratory fuels in vivo for postabsorptive rat small intestine. J Biol Chem 1978;253:69-76.
0 Rajas F, Bruni N, Montano S, Zitoun C, Mithieux G: The glucose- 6 phosphatase gene is expressed in human and rat small intestine: regulation of expression in fasted and diabetic rats. Gastroenterology 1999;117: 132-139.

11 Croset M, Rajas F, Zitoun C, Hurot JM, Montano S, Mithieux G: Rat small intestine is an insulin-sensitive gluconeogenic organ. Diabetes 2001;50:740-746.

12 Mithieux G, Bady I, Gautier A, Croset M, Rajas F, Zitoun C: Induction of control genes in intestinal gluconeogenesis is sequential during fasting and maximal in diabetes. Am J Physiol Endocrinol Metab 2004;286:E370E375.

13 Rajas F, Croset M, Zitoun C, Montano S, Mithieux G: Induction of PEPCK gene expression in insulinopenia in rat small intestine. Diabetes 2000;49:1165-1168.

14 Mithieux G, Vidal H, Zitoun C, Bruni N, Daniele N, Minassian C: Glucose-6 phosphatase mRNA and activity are increased to the same extent in kidney and liver of diabetic rats. Diabetes 1996;45:891-896.

15 Minassian C, Mithieux G: Differential time course of liver and kidney glucose- 6 phosphatase activity during fasting in rats. Comp Biochem Physiol 1994;109B:99-104.

16 Minassian C, Zitoun C, Mithieux G: Differential time-course of liver and kidney glucose- 6 phosphatase activity during long-term fasting in rat correlates with differential time-course of messenger RNA level. Mol Cell Biochem 1996;155:37-41.

17 Mithieux G, Daniele N, Payrastre B, Zitoun C: Liver microsomal glucose- 6 phosphatase is competitively inhibited by the lipid products of phosphatidyl-inositol 3-kinase. J Biol Chem 1998;273:17-19.

18 Daniele N, Rajas F, Payrastre B, Mauco G, Zitoun C, Mithieux G: Phosphatidylinositol 3-kinase translocates onto liver endoplasmic reticulum and may account for the inhibition of glucose- 6 phosphatase during refeeding. J Biol Chem 1999;274:3597-3601.

19 Mithieux G, Vega FV, Riou JP: The liver glucose-6-phosphatase of intact microsomes is inhibited and displays sigmoid kinetics in the presence of $\alpha$-keto glutarate-magnesium and oxalo-acetate-magnesium chelates. J Biol Chem 1990;265:20364-20368.

20 Mithieux G, Zitoun C: Mechanisms by which fatty acyl-CoA esters inhibit or activate glucose- 6 phosphatase in intact and detergenttreated rat liver microsomes. Eur J Biochem 1996;235:799-803.

21 Minassian C, Daniele N, Bordet JC, Zitoun C, Mithieux G: Liver glucose-6 phosphatase activity is inhibited by refeeding in rats. J Nutr 1995; 125:2727-2732.

22 Mithieux G, Rajas F, Gautier-Stein A: A novel role for glucose 6 phosphatase in the small intestine in the control of glucose homeostasis. J Biol Chem 2004;279:44231-44234.

23 Kirchner S, Seixas P, Kaushik S, Panserat S: Effect of low protein intake on extra-hepatic gluconeogenic enzyme expression and peripheral glucose phosphorylation in rainbow trout. Comp Biochem Physiol B 2005;140: 333-340.

24 Cui XL, Soteropoulos P, Tolias P, Ferraris RP: Fructose-responsive genes in the small intestine of neonatal rats. Physiol Genomics 2004; 18:206-217.

25 Yanez AJ, Nualart F, Droppelmann C, et al: Broad expression of fructose-1,6-bisphosphatase and phosphoenolpyruvate carboxykinase provide evidence for gluconeogenesis in human tissues other than liver and kidney. J Cell Physiol 2003;197:189-197.

26 Battezzati A, Caumo A, Martino F, Sereni LP, Coppa J, Romito R, Ammatuna M, Regalia E, Matthews DE, Mazzaferro V, Luzi L: Non hepatic glucose production in humans. Am J Physiol 2004;286:E124-E135. 
27 Tordoff MG, Tluczek JP, Friedman MI: Effect of hepatic portal glucose concentration on food intake and metabolism. Am J Physiol 1989;257:R1474-R1480.

-28 Langhans W, Grossmann F, Geary N: Intrameal hepatic-portal infusion of glucose reduces spontaneous meal size in rats. Physiol Behav 2001;73:499-507.

-29 Tordoff MG, Friedman MI: Hepatic portal glucose infusions decrease food intake and increase food preference. Am J Physiol 1986 251:R192-R196.

30 Niijima A: Glucose-sensitive afferent nerve fibres in the hepatic branch of the vagus nerve in the guinea-pig. J Physiol 1982;332:315323.

31 Adachi A, Shimizu N, Oomura Y, Kobashi M: Convergence of hepatoportal glucose-sensitive afferent signals to glucose-sensitive units within the nucleus of the solitary tract. Neurosci Lett 1984;46:215-218.

-32 Schmitt M: Influences of hepatic portal receptors on hypothalamic feeding and satiety centers. Am J Physiol 1973;225:1089-1095.

33 Shimizu N, Oomura Y, Novin D, Grijalva CV, Cooper PH: Functional correlations between lateral hypothalamic glucose-sensitive neurons and hepatic portal glucose-sensitive units in rat. Brain Res 1983;265:49-54.

34 Mithieux G, Misery P, Magnan C, Pillot B, Gautier-Stein A, Bernard C, et al: Portal sensing of intestinal gluconeogenesis is a mechanistic link in the diminution of food intake induced by diet protein. Cell Metab 2005;2: 321-329.
35 Delaere F, Duchampt A, Mounien L, Seyer P, Duraffourd C, Zitoun C, et al: The role of sodium-coupled glucose co-transporter 3 in the satiety effect of portal glucose sensing. Mol Metab 2012;2:47-53.

36 Penhoat A, Mutel E, Amigo-Correig M, Pillot B, Stefanutti A, Rajas F, et al: Protein-induced satiety is abolished in the absence of intestinal gluconeogenesis. Physiol Behav 2011;105:8993.

37 Berridge KC: 'Liking' and 'wanting' food rewards: brain substrates and roles in eating disorders. Physiol Behav 2009;97:537-550.

38 Zioudrou C, Streaty RA, Klee WA: Opioid peptides derived from food proteins. The exorphins. J Biol Chem 1979;254:2446-2449.

39 Capasso A, Amodeo P, Balboni G, Guerrini R, Lazarus LH, Temussi PA, et al: Design of mu selective opioid dipeptide antagonists. FEBS Lett 1997;417:141-144.

40 Moritoki H, Takei M, Kotani M, et al: Tripeptides acting on opioid receptors in rat colon. Eur J Pharmacol 1984;100:29-39.

41 Schiller P: Opioid dipeptide derivatives with a mixed $\mu$ antagonist $/ \delta$ antagonist, partial $\mu$ agonist $/ \delta$ antagonist or $\mu$ agonist/partial $/ \delta$ ag onist profile. American Peptide Symposia 2002, vol 6, pp 229-270.

42 Lee VH: Membrane transporters. Eur J Pharm Sci 2000; (suppl 2):S41-S50.

43 Duraffourd C, De Vadder F, Goncalves D, Delaere F, Penhoat A, Brusset B, et al: $\mathrm{Mu}$ opioid receptors and dietary protein stimulate a gut-brain neural circuitry limiting food intake. Cell 2012;150:377-388.

44 Mendeloff AI: Dietary fiber and human health. N Engl J Med 1977;297:811-814.

-45 De Vadder F, Kovatcheva-Datchary P, Goncalves D, Vinera J, Zitoun C, Duchampt A, et al: Microbiota-generated metabolites promote metabolic benefits via gut-brain neural circuits. Cell 2014;156:84-96.
Gautier-Stein A, Zitoun C, Lalli E, Mithieux G, Rajas F: Transcriptional regulation of the glucose-6-phosphatase gene by cAMP/vasoactive intestinal peptide in the intestine. Role of HNF4alpha, CREM, HNF1alpha, and C/ EBPalpha. J Biol Chem 2006;281:3126831278.

47 Sala PC, Torrinhas RS, Heymsfield SB, Waitzberg DL: Type 2 diabetes mellitus: a possible surgically reversible intestinal dysfunction. Obes Surg 2012;22:167-176.

48 Troy S, Soty M, Ribeiro L, Laval L, Migrenne $\mathrm{S}$, Fioramonti X, et al: Intestinal gluconeogenesis is a key factor for early metabolic changes after gastric bypass but not after gastric lapband in mice. Cell Metab 2008;8:201-211.

49 Hayes MT, Foo J, Besic C, Tychinskaya Y, Stubbs RS: Is intestinal gluconeogenesis a key factor in the early changes in glucose homeostasis following gastric bypass? Obes Surg 2011;21:759-762.

50 Mithieux G: Comment about intestinal gluconeogenesis after gastric bypass in human in relation with the paper by Hayes et al., Obes Surg 2011. Obes Surg 2011;22:1920-1922.

51 Sun D, Wang K, Yan Z, Zhang G, Liu S, Liu F, et al: Duodenal-jejunal bypass surgery upregulates the expression of the hepatic insulin signaling proteins and the key regulatory enzymes of intestinal gluconeogenesis in diabetic Goto-Kakisaki rats. Obes Surg 2013;23: 1734-1742.

52 Mithieux G: A synergy between incretin effect and intestinal gluconeogenesis accounting for the rapid metabolic benefits of gastric bypass surgery. Curr Diab Rep 2012;12:167-171. 